\title{
MARINA VOLONTÉ
}

\section{«Ebbi una passione spiccata per la storia». La figura di don Luigi Luchini e la donazione di materiali da Bedriacum al Museo Archeologico di Cremona}

\begin{abstract}
Nella storia delle ricerche archeologiche ottocentesche nel sito di Calvatone-Bedriacum riveste un ruolo centrale la figura di don Luigi Luchini, originario del Milanese e vissuto per gran parte della sua vita nel territorio della Diocesi di Cremona. Il Museo Civico Ala Ponzone conserva una serie di documenti d'archivio, interessanti per la ricostruzione dei primi ritrovamenti nel vicus e delle vicissitudini della collezione che da essi ebbe origine.
\end{abstract}

Parole chiave - Luigi Luchini; Bedriacum; collezione; Museo Archeologico di Cremona; archivio

Title - «I had a keen strong passion for history». The figure of Father Luigi Luchini and the donation of materials from Bedriacum to the Archaeological Museum of Cremona

Abstract - In the history of the 19th-century archaeological research in Calvatone-Bedriacum, Father Luigi Luchini, born in Milan but lived for most of his life in the territory of the Diocese of Cremona, played a central role. The Ala Ponzone Civic Museum keeps interesting archival documents to reconstruct the first findings of the vicus and the vicissitudes of the collection that originated from them.

Keywords - Luigi Luchini; Bedriacum; collection; Archaeological Museum of Cremona; archive

Don Luigi Luchini ${ }^{1}$ è ben noto nella storia degli studi ${ }^{2}$ su Bedriacum grazie al suo Bebriaco illustrato dai suoi scavi archeologici. Prima pagina di storia cremonese. Memoria del parroco Luigi Luchini, edito a Casalmaggiore nel $1878^{3}$. A fronte delle molte citazioni di questo studio, non è mai stata dedicata particolare attenzione alla ricerca di notizie sulla figura dell'autore. Alcune indicazioni biografiche sono contenute nel catalogo annotato che censisce per la prima volta i manoscritti della Biblioteca del Seminario di Cremona ${ }^{4}$, redatto anch'esso in via manoscritta nel 1933 dal sacerdote Felice Zanoni, all'epoca seminarista.

\footnotetext{
${ }^{1}$ Il cognome si trova scritto nei documenti con una o due "c".

${ }^{2}$ L'elenco esaustivo delle pubblicazioni dell'Università degli Studi di Milano si trova sul sito del "Progetto Calvatone": https://progettocalvatone.unimi.it/pubblicazioni.html, a cui va aggiunta l'edizione degli scavi della Soprintendenza nel "Campo del Generale", diretti da Lynn Pitcher (Bedriacum 1996).

${ }^{3}$ La pubblicazione riscosse ai tempi dell'autore notevole interesse. Tra le recensioni conservate dal Luchini medesimo (si veda alla nota successiva) si trova per esempio quella pubblicata ne Lo spettatore in data 8-9 ottobre 1878, in cui il testo è definito «chiaro e spigliato ne lo stile, ed anche vivace e lepido così da animare una materia per i profani arida e pesante».

${ }^{4}$ La Biblioteca possiede diversi documenti relativi a Luigi Luchini, che mi sono stati resi noti dalle bibliotecarie Roberta Aglio e Monica Feraboli, che ringrazio per il prezioso aiuto. Si tratta di una coperta in pelle di altro volume, riutilizzata per raccogliere del materiale eterogeneo del sacerdote; sul piatto superiore è posta un'etichetta con la scritta autografa di Luchini barrata (a scopo di cancellarla) «Luigi Luchini / Ricordi di mia vita», sostituita con la scritta non autografa «Giudizi della stampa / sulle opere di Luchini». All'interno si trovano diversi ritagli di quotidiani o periodici relativi alle opere di Luchini (recensioni, lettere ai giornali), con un piccolo commento dell'autore; ci sono anche diversi biglietti da visita di personalità coeve, come Cesare Cantù e sono incollate tra le pagine anche delle lettere. C'è poi una cartella nel Fondo Manoscritti, con la collocazione Ms. 112, contenente cinque fascicoli, ognuno contenente degli appunti scritti dallo stesso Luchini su diversi argomenti; il terzo fascicolo, donato al Seminario dal sacerdote Giuseppe Boni, porta il titolo Ricordi di mia vita ed è formato da 15 fogli autografi di Luchini; qui, alla prima pagina del paragrafo XV Le mie Opere Storiche. Relativi Onori e i Commenti della
} 
Da esse apprendiamo che il Luchini nacque a Cernusco sul Naviglio il 22 febbraio $1838^{5}$, fu alunno del Seminario di Milano e accolto nella Diocesi di Cremona nel 1860, dove fu ordinato sacerdote l'anno seguente dal vescovo Novasconi; fu vicario a Cella Dati e Cortetano, per poi passare come parroco nel 1870 ad Acqualunga Badona e [dal 1876] a Romprezzagno; fu anche nominato "Cavaliere e Regio Ispettore dei Monumenti"; morì nel 1911, il 5 settembre, pochi giorni dopo la celebrazione della messa d'oro, corrispondente al cinquantesimo anno dall'ordinazione sacerdotale.

Il periodo trascorso a Romprezzagno fu quello in cui don Luigi poté dar corso alla sua passione per l'antichità, non solo dedicandosi al sopra citato studio storico su Bedriacum, ma anche raccogliendo oggetti provenienti dal sito.

Come egli stesso riferisce ${ }^{7}$, infatti, fu solo quando fece ingresso nella parrocchia di Romprezzagno che gli «si offrì il movente», nel momento in cui gli vennero presentate «anticaglie estratte dai contadini dagli scavi delle aree di S. Andrea».

Come vedremo, delle "anticaglie" in possesso di don Luigi esiste una documentazione varia, anche se incompleta; una parte di esse, essendo stata donata al Museo di Cremona, è ben conosciuta e studiata.

La consistenza della donazione ci è nota dall'inventario manoscritto Rubrica S. Materiale di Scavo, compilato dal Conservatore Antonio Sommi Picenardi e da Carlo Crippa, custode, con data 1914, nella sezione Scavi in Provincia. Supposto Bebriaco, Seniga, Calvatone ed altri siti con provenienza «Sac. D. Luigi Lucchini» o «Dono D. Luigi Luchini». Le altre provenienze citate nella sezione bedriacense sono una donazione di Vincenzo Bonvini, a volte citato con la sorella, due oggetti singoli ricevuti rispettivamente dal «Sac. Prof. Pizzi» e dal Prof. Davide Bergamaschi, nonché il Municipio di Cremona, a cui segue quasi sempre la corrispondenza con il numero di inventario del Museo Provinciale e pertanto con ogni probabilità provenienti nella loro totalità da tale raccolta .

Gli oggetti che risultano donati da don Luchini sono una trentina, oltre a un nucleo di 167 monete in argento e bronzo ${ }^{10}$; i materiali sono soprattutto relativi all'instrumentum metallico e alla ceramica, con qualche attestazione di appliques e piccole sculture in bronzo e anche in marmo ${ }^{11}$. Risulta

\footnotetext{
Stampa, si trova la frase citata nel titolo «... ebbi una spiccata passione per la storia».

${ }^{5}$ Egli si dedicò anche a studi storici sul suo paese di origine, come si evince dalla citazione in un volume di Luigi Ghezzi relativo alle memorie storiche di Cisnusculum: GHEZZI 1911, p. 6.

${ }^{6}$ Così scrive esattamente Zanoni, anche se la carica era generalmente denominata "Regio Ispettore degli Scavi e Monumenti".

${ }^{7}$ Vedi alla nt. 3, Ricordi di mia vita.

${ }^{8}$ L'ubicazione dell'antica Bedriacum (indicata nell'Ottocento, anche dal Luchini medesimo, come Bebriaco) nei pressi dell'attuale Calvatone è stata data pressoché per certa a partire dagli studi di Giuseppe Patroni: si veda PASSI PITCHER 1991, p. 41. Per una raccolta delle diverse collocazioni del sito, si veda PONTIROLI 1972, p. 90, nt. 3.

${ }^{9}$ Il Museo Provinciale era nato nel 1877 dall'intento della Regia Commissione Provinciale per la conservazione dei monumenti di non disperdere oggetti di valore storico e storico-artistico provenienti dal territorio; si veda VOLONTÉ 2009, p. 23.

${ }^{10}$ Come accade di frequente per le collezioni numismatiche di formazione ottocentesca, anche nel Medagliere del Museo Civico di Cremona non sempre sono reperibili le indicazioni di provenienza delle monete; questo è purtroppo il caso di quelle donate da Luchini.

${ }^{11}$ Gli oggetti che erano esposti nel vecchio allestimento della Sezione Archeologica del Museo al piano terra di Palazzo Affaitati (ora sostituita dal Museo Archeologico nella chiesa sconsacrata di San Lorenzo) sono in primis pubblicati nel relativo catalogo (PONTIROLI 1974).
} 
appartenente al nucleo anche il corpo di fibula a sanguisuga ${ }^{12}$, unica traccia nota di una presenza preromana del sito, oltre alla sepoltura dell'Età del Rame rinvenuta sotto la domus del Labirinto ${ }^{13}$.

Pur essendo completamente privi di dati puntuali sul rinvenimento, i materiali, studiati nell'ambito delle relative classi tipologiche e funzionali, contribuiscono in maniera significativa alla conoscenza della storia del vicus e pertanto se ne richiamano in questa sede i principali.

Probabilmente legata al culto domestico è la statuetta acefala in marmo bianco raffigurante la dea Igea $^{14}$, identificabile grazie alla presenza del serpente stretto nella mano destra, di fattura assai accurata. La scultura, databile al II sec. d.C., è attualmente esposta al Museo Archeologico di Cremona allestito dal 2009 nella chiesa sconsacrata di San Lorenzo. La statuetta di Asclepio, altra divinità salutifera, apparentemente non presente nella Rubrica e documentata da disegni ${ }^{15}$, non è invece rintracciabile.

All'ambito dell'arredo domestico ${ }^{16}$ appartengono anche le tre appliques in bronzo raffiguranti busti di divinità (Giove, Minerva e un personaggio giovanile già identificato con Eros), interpretabili come elementi metallici decorativi di casse in legno; ancora a un arredo domestico, oppure alla decorazione di un carro $^{17}$, è pertinente un gruppo raffigurante Mercurio con un ariete e un gallo, derivante da tipi statuari tardoclassici.

Per quanto riguarda la ceramica, fanno parte con certezza della collezione una coppetta in terra sigillata di forma Drag $24 / 25^{18}$ e due lucerne ${ }^{19}$.

Interessante per la rarità di ritrovamenti di questo tipo nei contesti bedriacensi è il nucleo di oggetti di ambito militare ${ }^{20}$, in particolare un tribulus, una punta di lancia e una punta di freccia, oltre a un bullone per catapulta, recentemente prestati alla mostra londinese su Nerone ${ }^{21}$ ed esposti a simboleggiare le lotte per la conquista del potere imperiale seguite alla sua morte.

Oltre agli oggetti descritti, il Museo Civico Ala Ponzone conserva interessanti documenti d'archivio, in particolare album di disegni ${ }^{22}$, in cui è fatto esplicito riferimento alla collezione di Luigi Luchini.

In uno degli album ${ }^{23}$ compaiono la statuetta di Igea ( Statua in marmo pentelico, mancante del capo, trovata a S. Andrea, ora posseduta dall'Egr. ${ }^{\circ}$ Parroco D. Luigi Lucchini»), una altrimenti ignota terracotta («Mascherone da teatro disegnato a grandezza vera, lavoro in terra cotta trovato a S. Andrea, ora è presso all' Egr. ${ }^{\circ}$ D. Lucchini») e due balsamari («fiale lagrimatorie») (Fig. 1), uno in vetro e l'altro in ceramica, non identificabili tra gli oggetti conservati in museo; nelle ultime pagine, vi sono le «Fotografie rappresentanti tutti i preziosi oggetti di scavo trovati a S. Andrea di Calvatone (Bebriacus) e

\footnotetext{
${ }^{12}$ ARSLAN 2003, p. 64.

${ }^{13}$ PeARCE 2003, p. 50.

${ }^{14}$ BAIGUERA 2009.

${ }^{15}$ In LUCHINI 1878 e nell'album nr 115, senza però una didascalia che ricolleghi il pezzo alla collezione Luchini.

${ }^{16}$ Gli elementi di arredi in metallo sono stati studiati da Marina Castoldi: CASTOLDI 2005, pp. 189-191 e p. 200 , nn. 2, 3, 4, 5.

${ }^{17}$ BOLLA 1997, p. 15.

${ }^{18}$ Inv. MC 1291: CORSANO 1990, p. 55, nr. C68, tav. IV, 2.

${ }^{19}$ Pontiroli 1980, nn. 26 e 36.

${ }^{20}$ I militaria da Calvatone furono pubblicati da Emanuela Natta nel 1996: PASSI PITCHER - NATTA - SARTORI 1996, pP. $123-$ 125.

${ }^{21}$ OpPer 2021, p. 273.

${ }^{22}$ Già citati in relazione allo studio delle collezioni museali (VOLONTÉ 2009, p. 27) nonché alla documentazione sul ritrovamento della Vittoria di Calvatone (VOLONTÉ 2015, pp. 41-43).

${ }^{23}$ Archivio Museo Civico Ala Ponzone, Archeologia, nr. 114.
} 
che ora sono posseduti dall'Egregio Preposto di Romprezzagno R. ${ }^{\circ}$ D.n Luigi Lucchini, autore dell'opera Bebriaco illustrato che si unisce ai presenti disegni».

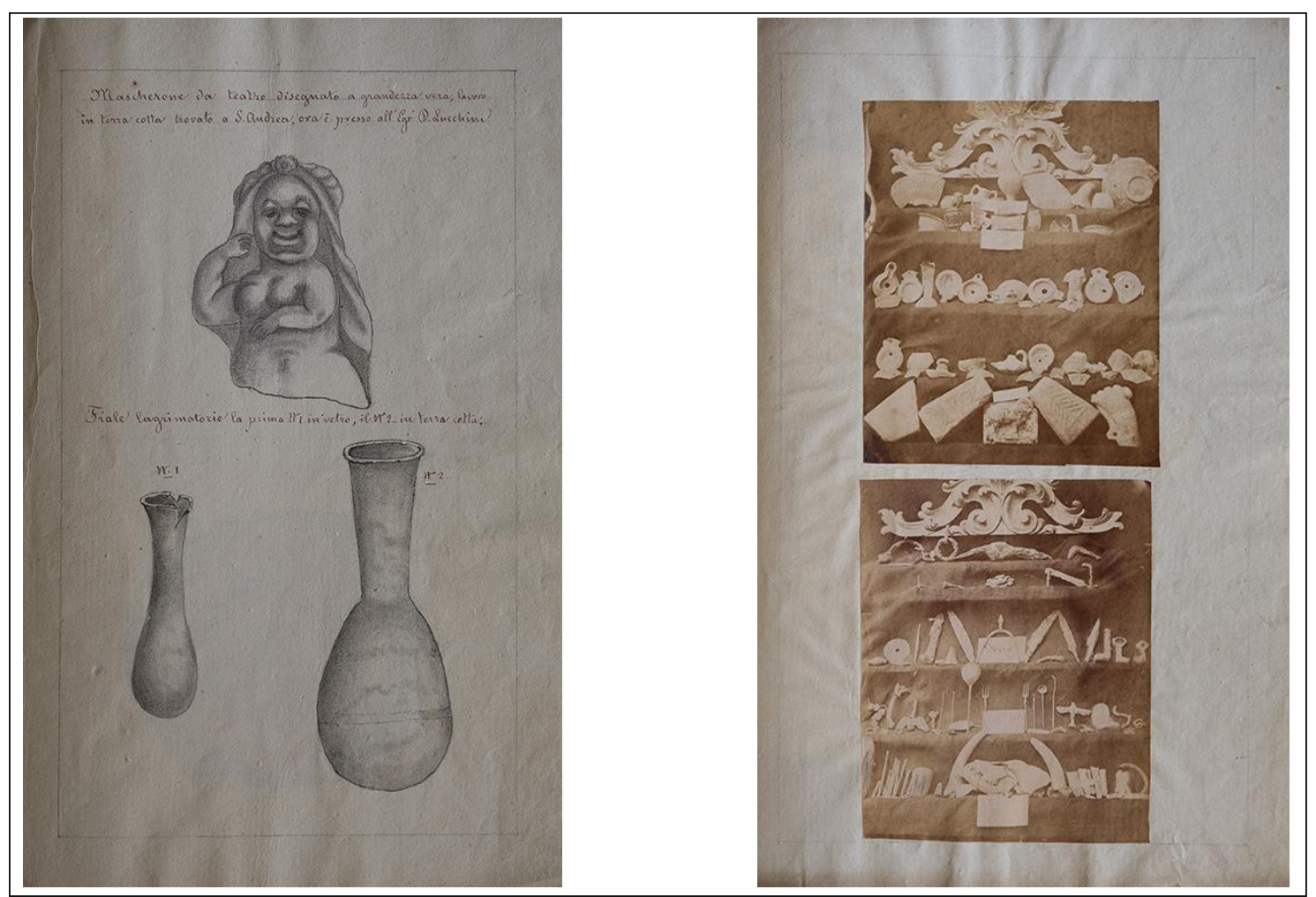

Figg. 1-2. Archivio Museo Civico Ala Ponzone, Archeologia, pagine dell'album nr. 114.

Le fotografie (Fig. 2) ritraggono oggetti in parte corrispondenti a quelli disegnati (lucerne, pesi da telaio, un'anfora, cucchiai e forchette...), mentre altri li troviamo per la prima volta: in particolare due olle con una brocca e un gruppo di esagonette pavimentali.

In un altro album ${ }^{24}$ una tavola raffigura alcuni frammenti di ceramica a rilievo (Fig. 3), tra cui un'olletta antropoprosopa, insieme a una «testina di marmo molto guasta» e a «un pezzo di vetro lavorato e smaltato bleu», un'altra (Fig. 4) un «frammento di statuetta di terra cotta verniciata in rosso»e un tappo d'anfora con lettere in rilievo, definito «coperchio di terra cotta di un'Olla Cineraria a caratteri sconosciuti», le ultime due (che evidentemente non riguardano materiali solo di epoca romana) alcuni cucchiai, forchette (Fig. 5) e un oggetto definito «falcetto».

Sono inoltre presenti nell'archivio due elenchi ${ }^{25}$ parziali di oggetti da Calvatone «donati da don Lucchini» (tra cui solo alcuni riconducibili alle descrizioni nell'inventario) e una raccolta di fotografie descritte da Giuseppe Pontiroli ${ }^{26}$ come «di materiale probabilmente di proprietà del sac. Giovanni [sic] Lucchini di Romprezzagno facenti parte di una sua raccolta privata» ${ }^{27}$ : esse ritraggono in realtà una

\footnotetext{
${ }^{24}$ Archivio Museo Civico Ala Ponzone, Archeologia, nr. 116.

${ }^{25} \mathrm{Nr} .121$ e 162

${ }^{26}$ A cui si deve un primo ordinamento dell'archivio cartaceo, poi oggetto di sistemazione e catalogazione da parte di Emilio Giazzi nel 1995.

${ }^{27} \mathrm{Nr}$. 170. Il nome Giovanni è evidentemente un errore; le foto ritraggono oggetti certamente parte della raccolta di don Luigi, come la statuetta di Igea.
} 
sorta di collage di disegni (Fig. 6), in cui si riconoscono pezzi presenti in museo o noti dai documenti sopra citati (ancora una volta le statuette di Igea e Asclepio, alcune lucerne tra cui tuttavia non si riconoscono le due conservate al museo, pesi da telaio iscritti, un'anfora) insieme ad altri (tra cui rilievi ed iscrizioni) non riscontrabili e talora difficilmente interpretabili.

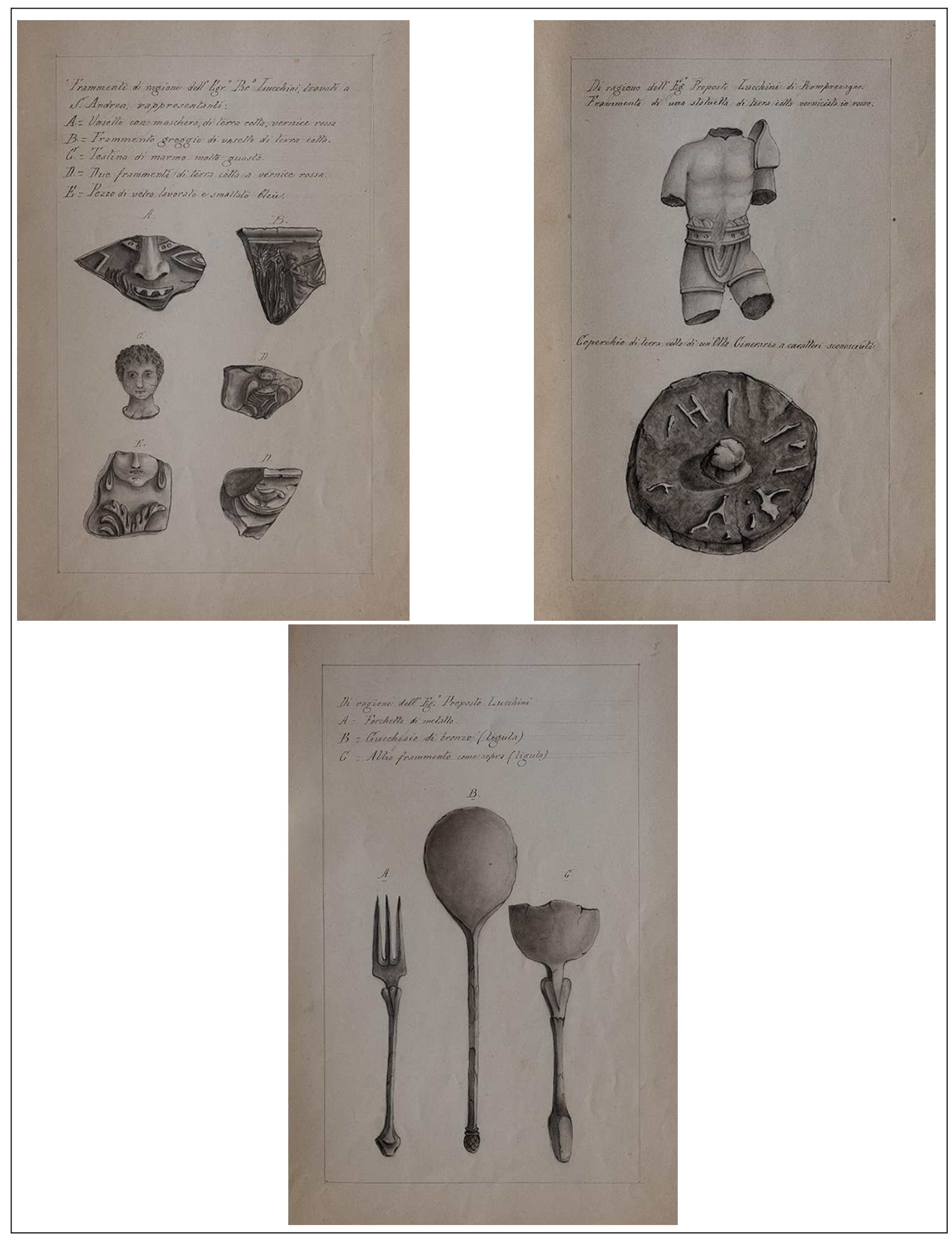

Figg. 3-5. Archivio Museo Civico Ala Ponzone, Archeologia, pagine dell’album nr. 116. 


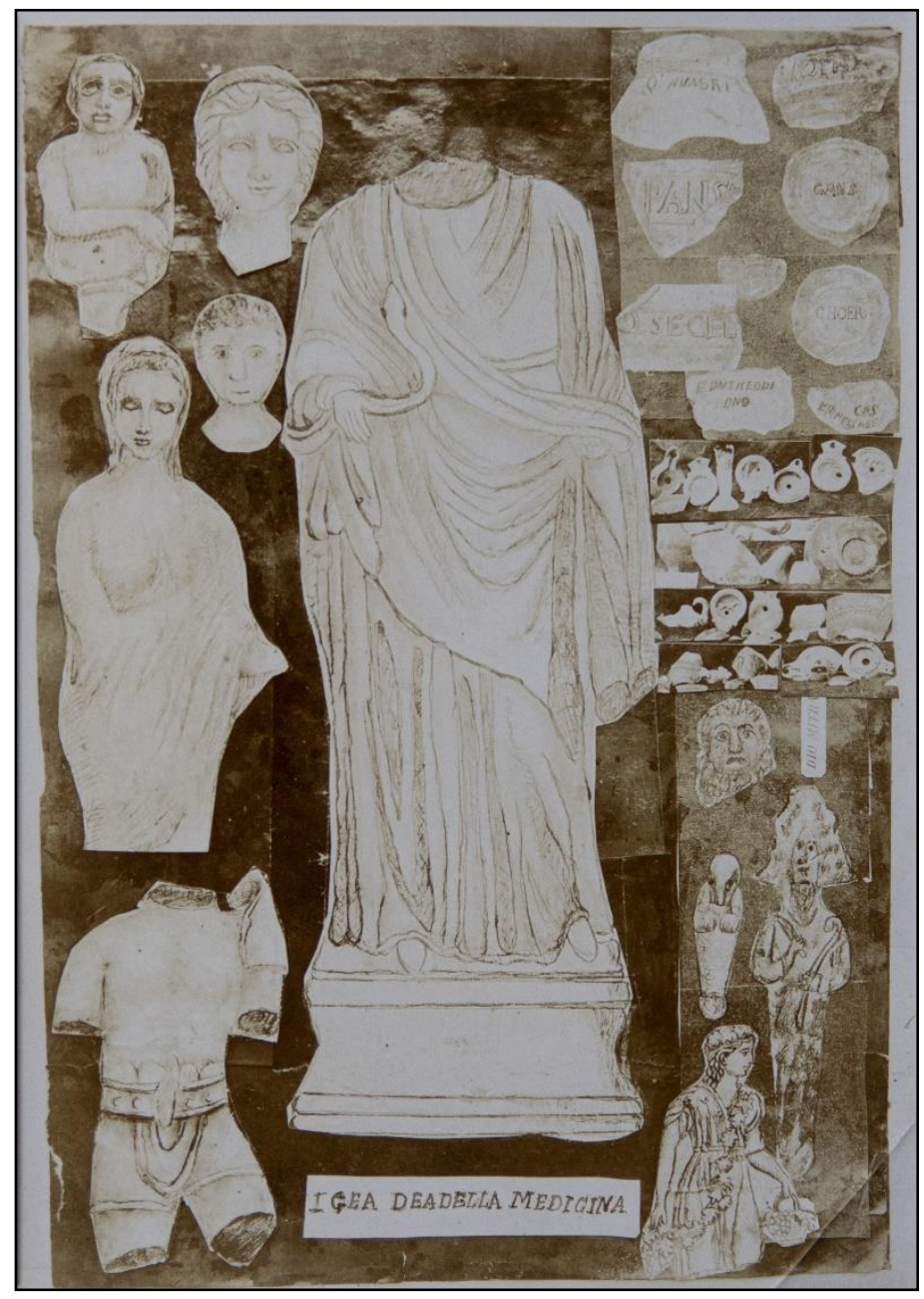

Fig. 6. Archivio Museo Civico Ala Ponzone, Archeologia, fotografia di collage di disegni.

Da questa documentazione appare chiaro che non tutti i materiali posseduti dal Luchini furono consegnati alle pubbliche raccolte. A complicare la questione, sappiamo che nel 1879, una decina di anni prima, quindi, della costituzione e apertura del Civico Museo (1888), don Luigi tentò di vendere al Municipio l'intera collezione da lui posseduta ${ }^{28}$.

Ecco quanto scrive il Luchini: «Qualora la Commissione domandasse un sussidio straordinario al Municipio, io cederei la mia collezione archeologica a stima di intelligenti, per prezzo da convenirsi, e avrei caro si conservasse nella Provincia ciò che è suo»; in calce alla lettera, Francesco Robolotti ${ }^{29}$ annota la risposta negativa della commissione del Regio Museo Provinciale alla proposta.

Il fatto che negli inventari del Museo Civico $^{30}$ sia fatto esplicito riferimento a un dono, significa evidentemente che il Luchini, alcuni anni dopo la tentata vendita decise di conferire una parte della

\footnotetext{
${ }^{28}$ Archivio Museo Civico Ala Ponzone, Archeologia, nr. 111.

${ }^{29}$ Francesco Robolotti (1802-1885) fu medico presso l'ospedale maggiore di Cremona, patriota e appassionato studioso di storia, soprattutto medievale.

${ }^{30} \mathrm{Si}$ veda supra.
} 
raccolta alla nuova istituzione, trattenendo invece alcuni oggetti, di cui non siamo in grado di seguire le vicissitudini ${ }^{31}$.

Comunque sia, i materiali della raccolta bedriacense furono oggetto di costante interesse e approfondimento da parte del Luchini, come dimostra ad esempio uno scambio di missive tra il sacerdote e il conservatore del museo Carlo Crippa, relativa alle «piramidette» (i pesi da telaio) in terracotta $^{32}$. Lo stesso Crippa a sua volta si rivolge al Luchini chiedendogli un parere sulla possibile provenienza da Calvatone di «molti frammenti di intonaco di calce dipinti a fresco a vari colori a linee e a fiori, dell'epoca romana» ${ }^{33}$.

Un altro documento interessante, che documenta l'attività di don Luigi anche al di fuori del sito di Calvatone, è la Lettera di Carlo Crippa custode del Museo 'A. Ponzone' nella quale avverte il Sindaco di Cremona che il parroco di Romprezzagno don Luigi Lucchini ha donato al Museo oggetti di scavo provenienti da Vho di Piadena, datata 19 maggio $1890^{34}$. Nella lettera, si fa riferimento a «27 piccoli pezzi di lavori litici diversi» trovati «nel fondo detto Costiera lungo la via Mantovana», elencati in forma più dettagliata nella Rubrica $\mathrm{S}^{35}$.

Don Luigi Luchini non è l'unica figura di sacerdote attivo nel campo della ricerca archeologica nel territorio tra Cremona e Mantova tra la seconda metà del XIX secolo e gli inizi del XX.

Ricordiamo l'opera di don Gioachino Bonvicini (1849-1929), autore del manoscritto Memorie storiche di Ognissanti conservato presso la Biblioteca del Seminario Vescovile di Cremona ${ }^{36}$ che riporta importanti notizie e rilievi, sia dell'area di scavo, sia dei materiali recuperati nel sito della terramara ubicata nel territorio del Comune di Cella Dati; o ancora l'opera di don Antonio Parazzi (1823-1899), arciprete di Viadana, autore di Origine e vicende di Viadana e del suo distretto, che fu nominato Ispettore agli Scavi e ai Monumenti di Antichità e fu fondatore del Museo di Viadana, a lui dedicato ${ }^{37}$. Un ruolo fondamentale per l'archeologia del Cremonese fu poi rivestito da Francesco Pizzi, membro della Regia Commissione Provinciale per la conservazione dei Monumenti, a cui si deve l’istituzione del più volte citato Museo Provinciale.

La trascrizione e lo studio dei manoscritti di questi importanti testimoni, e protagonisti, dell'archeologia territoriale fanno parte di un lavoro di ricerca in corso $^{38}$, di cui il presente contributo costituisce un primo approfondimento.

Marina Volonté marina.volonte@,comune.cremona.it

\footnotetext{
${ }^{31}$ Anche Giuseppe Pontiroli, allora Conservatore onorario del Museo, in una nota datata marzo 1971 allegata all'album nr. 115, scrive che il documento «ha permesso di identificare materiali giunti in Museo, parte esposti e parte in magazzino. Altri non sono pervenuti o sono andati perduti».

${ }^{32}$ Archivio Museo Civico Ala Ponzone, Archeologia, nr. 208 e 210.

${ }^{33}$ Archivio Museo Civico Ala Ponzone, Archeologia, nr. 205.

${ }^{34}$ Archivio Museo Civico Ala Ponzone, Archeologia, nr. 156.

${ }^{35}$ Alla p. 12.

${ }^{36}$ Si veda in proposito VOLONTÉ 2019, pp. 24-26.

${ }^{37}$ Benedetti - Morandini - Volonté 2012, pp. 18-21. Nel fascicolo del Fondo Manoscritti della Biblioteca del Seminario Vescovile, già citato, Ricordi di mia vita (MS. 112.3), Luchini scrive tra l'altro un paragrafo (XVI) denominato Le ombre di alcuni amici letterati, passati attraverso la lanterna magica, in cui cita don Antonio Parazzi che gli si presenta con una «borsa di cocci frantumati trovati nei suoi fondi parrocchiali».

${ }^{38}$ La ricerca è stata avviata a seguito del riconoscimento dei manoscritti del Bonvicini nella Biblioteca del Seminario Vescovile, la cui trascrizione è in corso da parte di Roberta Aglio e Monica Feraboli.
} 


\section{Abbreviazioni bibliografiche}

\section{ARSLAN 2003}

E.A. Arslan, La seconda età del ferro nel territorio dell'attuale provincia di Cremona, in Storia di Cremona 2003, pp. 62-83.

\section{BAIGUERA 2009}

E. Baiguera, Statuetta di Igea, in Sculture 2009, pp. 103-107.

\section{Bedriacum 1996}

L. Passi Pitcher (a cura di), Bedriacum. Ricerche archeologiche a Calvatone, Milano 1996.

BENEDETTI - MORANDINI - VOLONTÉ 2012

D. Benedetti - F. Morandini - M. Volonté, Dalla scoperta del territorio ai musei moderni. Storia delle ricerche, in Archeologia della Lombardia Orientale. I musei della Rete $M A \_$net e il loro territorio, Firenze 2012, pp. 11-21.

\section{BOLLA 1997}

M. Bolla, Bronzi figurati romani nelle Civiche Raccolte Archeologiche di Milano, Milano 1997 (Rassegna di Studi del Civico Museo Archeologico e del Civico Gabinetto Numismatico di Milano, Suppl. XVII).

\section{CASTOLDI 2005}

M. Castoldi, Cremona e l'ager Cremonensis: elementi d'arredo in bronzo, in F. Slavazzi (a cura di), Arredi di lusso di età romana. Da Roma alla Cisalpina, Firenze 2005 (Flos Italiae. Documenti di archeologia della Cisalpina Romana, 6), pp. 187-207.

\section{CORSANO 1990}

M. Corsano, Materiali da Calvatone. La raccolta del Museo di Cremona e lo scavo del pozzo del mappale $n$. 50, in "Rivista Archeologica Comense" 172 (1990), pp. 7-85.

\section{GHEZZI 1911}

L. Ghezzi, Cisnusculum: memorie storiche relative a Cernusco sul Naviglio, Monza 1911.

\section{LUCHINI 1878}

L. Luchini, Bebriaco illustrato dai suoi scavi archeologici. Prima pagina di storia cremonese. Memoria del parroco Luigi Luchini, Casalmaggiore (Cr) 1878.

OPPER 2021

T. Opper, Nero the man behind the myth, London 2021.

\section{PASSI PITCHER 1991}

L. Passi Pitcher, Storia degli scavi, in G.M. Facchini (a cura di), Calvatone romana. Studi e ricerche preliminari, Milano 1991 (Quaderni di ACME, 13), pp. 39-49.

PASSI PITCHER - NATTA - SARTORI 1996 L. Passi Pitcher - E. Natta - A. Sartori, I materiali dei vecchi rinvenimenti, in Bedriacum 1996, pp. 119-128.

PEARCE 2003

M. Pearce, Una pianura tra le acque: preistoria e protostoria del Cremonese, in Storia di Cremona 2003, pp. 38-61.

\section{PONTIROLI 1972}

G. Pontiroli, Gli scavi di Bedriacum nell'Ottocento, in Archeologia e storia nella Lombardia Padana. Bedriacum nel XIX centenario delle battaglie, Atti del convegno (Varenna 1969), Como 1972, pp. 89101.

\section{PONTIROLI 1974}

G. Pontiroli, Catalogo della Sezione Archeologica del Museo Civico "Ala Ponzone", Milano 1974.

PONTIROLI 1980

G. Pontiroli, Lucerne antiche dei musei di Cremona, Milano 1980.

Sculture 2009

F. Slavazzi - M. Volonté (a cura di), Sculture, materiali architettonici e di arredo delle raccolte archeologiche di Cremona, Milano 2009 (Corpus 
Signorum Imperii Romani. Italia. Regio $X$. Cremona).

Storia di Cremona 2003

P. Tozzi (a cura di), Storia di Cremona. L'Età Antica, Azzano San Paolo (Bg) 2003.

VOLONTÉ 2009

M. Volonté, Marmi e terracotte nelle raccolte del Museo Archeologico, in Sculture 2009, pp. 17-32.

VOLONTÉ 2015

M. Volonté, Le vicende ottocentesche: la Vittoria "senza ale", in F. Cacciatori - M. Volonté (a cura di), 1937. La Vittoria alata e le celebrarioni Stradivariane, Cremona 2015, pp. 37-44.

VOLONTÉ 2019

M. Volonté, Dalla preistoria alla fine dell'età romana, in F. Ghisolfi - G. Scotti (a cura di), Cella Dati storia e territorio, Castelleone (Cr) 2019, pp. 21-31. 